\title{
Open Resonator System for Reflectarray Elements Characterization
}

\author{
G. Di Massa, S. Costanzo, and O. H. Moreno \\ Dipartimento di Elettronica, Informatica e Sistemistica, Universitá della Calabria, 87036 Rende, Italy \\ Correspondence should be addressed to S. Costanzo, costanzo@deis.unical.it
}

Received 29 February 2012; Accepted 5 June 2012

Academic Editor: Manuel Arrebola

Copyright () 2012 G. Di Massa et al. This is an open access article distributed under the Creative Commons Attribution License, which permits unrestricted use, distribution, and reproduction in any medium, provided the original work is properly cited.

An open resonator system is proposed in this work to study the phase behavior of reflectarrays cells. The characterization of the single reflectarray element is performed by assuming a Gaussian plane wave normally incident on an infinite periodic array of identical radiating elements. An equivalent circuit model is properly derived for the open cavity including the reflectarray test structure, in order to retrieve the phase design curve of the single reflectarray element. K-band experimental validations are presented and discussed to show the effectiveness of the approach.

\section{Introduction}

Printed reflectarray antennas are low profile reflectors consisting of a planar array of microstrip patches illuminated by a feed [1-6]. Each reflectarray element is designed to compensate for the phase delay due to the relative feed-element path and to properly reradiate the incident field for obtaining a prescribed beam shape and direction. Reflectarray antennas inherit all appealing features of microstrip technology, such as less weight, easy manufacturing, and low cost, so revealing useful in many application fields such as remote sensing and satellite communications, millimeter wave [7], and infrared frequencies [8].

Many different phase tuning methods have been introduced in the literature for microstrip reflectarray antennas. These include variable size microstrip patches [9], variable size dipoles, and identical patches elements having variable length phase delay lines with or without angular rotations $[10,11]$.

Recently, phase-tunable elements have been considered to give reflectarrays with a steerable main beam [12-14]. These configurations, combined with dedicated electronic control systems and ad-hoc synthesis algorithm [15], are able to provide extremely versatile reconfigurable antennas [1618].
To successfully perform the design of a microstrip reflectarray, the characterization of the field reflected by the single radiating element embedded on the whole reflecting surface is of primary importance. As a matter of fact, the behavior of the reflected phase versus frequency and/or tuning element variation is used as design curve in the synthesis algorithm [15] to properly choose those elements that are able to give the prescribed reflectarray beam features.

The measurement of phased-array antennas in waveguide technology is a well-established technique [19]. In [20, 21], an equivalent waveguide approach is proposed for reflectarrays of variable size patches, with the unit cell enclosed in a rectangular waveguide having perfect electric and magnetics walls.

In $[22,23]$, an equivalent electrical circuit is adopted for the reflectarray, in order to derive the input reflection coefficient. In [22], the analysis is done in the terahertz frequencies band. The reflected phase is directly computed from the input reflection coefficient of a plane wave at the interface between the substrate and the air transmission layers. The input impedance of the reflecting cell is found in terms of the parallel between the patch impedance and the input impedance of the transmission line which is terminated into a short circuit. In [23], a resonant LC parallel circuit is adopted to model the reflectarray unit cell. Losses 
TABle 1: Expressions for the circuit elements of Figure 2.

\begin{tabular}{cccc}
\hline$L_{o}$ & $\mu_{0} l(\mathrm{H})$ & $C$ & $\epsilon l /\left(k_{\mathrm{o}} l\right)^{2}(\mathrm{~F})$ \\
$L^{\prime}$ & $\mu_{0} \delta(\mathrm{H})$ & $R^{\prime}$ & $2 / \sigma \delta(\Omega)$ \\
$L_{e}$ & $16.510^{-3} \mu_{0} a(\mathrm{H})$ & & \\
\hline
\end{tabular}

are taken into account by placing a resistor in series with the capacitor. The analysis is performed at $10 \mathrm{GHz}$. In [24], two resonant LC series circuits are considered instead of the simple resonant LC parallel circuit discussed in [23]. Four frequencies are considered, two resonant and two antiresonant.

In this paper, an open resonator [25] system is considered to characterize the phase response of variable size reflectarray patch elements. At this purpose, a full equivalent circuit is derived to include both the open resonator as well as the grounded reflecting surface, and the phase behavior of the field reirradiated by the reflectarray element is simply derived from the resonance behavior of the assumed equivalent circuit. K-band experimental validations are discussed to prove the validity of the proposed approach.

\section{Equivalent Circuit Description}

The standard configuration of an open cavity, composed by two spherical mirrors at distance $2 l$ and coupled with a rectangular waveguide, is reported in Figure 1. This structure has been studied in details in literature [26], thus confirming that the coupling between the cavity modes is negligible, so that the single resonant mode approximation gives a good description of the cavity field as only this last mode is practically excited. At the same time, the analysis of the solutions has also demonstrated that the excitation of higherorder waveguide modes $\mathrm{TE}_{n 0}$ is important for the accurate coupling description.

The equivalent circuit, adopted in [26] to accurately model an empty cavity coupled to a feeding waveguide, is illustrated in Figure 2.

The explicit expressions of the circuit elements as a function of the cavity and the waveguide parameters are reported in Table 1. In particular, the component $R^{\prime}$ models the losses due to the finite conductivity of the mirrors, while the term $L^{\prime}$ gives the effect of the skin depth $\delta$. It sums to the inductive circuit part $L_{o}$ modelling the cavity, thus producing a shift in the resonant frequency, which is equivalent to a cavity enlargement. The $L_{o}, C$ series models the resonant cavity, while the component $L_{e}$ takes into account the effect of cutoff modes in the feeding waveguide. The term $\beta_{01}$ gives the waveguide-cavity factor as reported in [26], and $Z_{g}$ is the characteristic impedance of feeding rectangular waveguide excited in its fundamental mode $\mathrm{TE}_{10}$.

In Table 1 , the term $2 l$ gives the cavity length, $a$ is the major waveguide dimension, $\sigma$ represents the conductivity, while $k_{o}=2 \pi f_{o} / c$ is the free-space propagation constant, with $c$ being the velocity of light and $f_{o}$ the resonant frequency of the empty resonator.

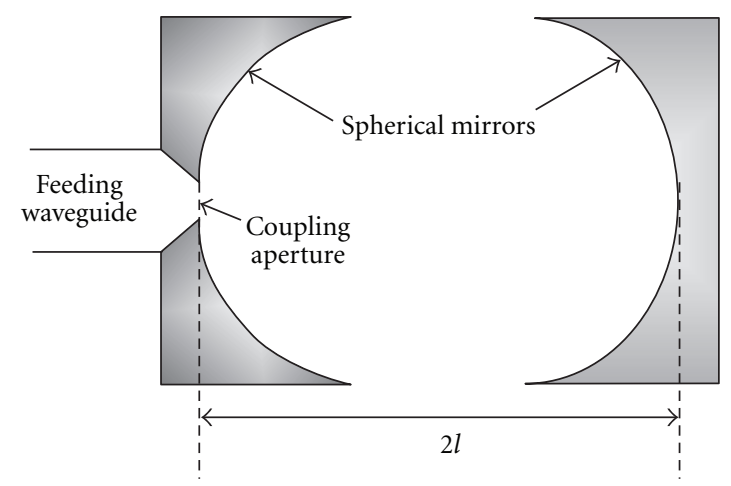

Figure 1: Open cavity fed by a rectangular waveguide.

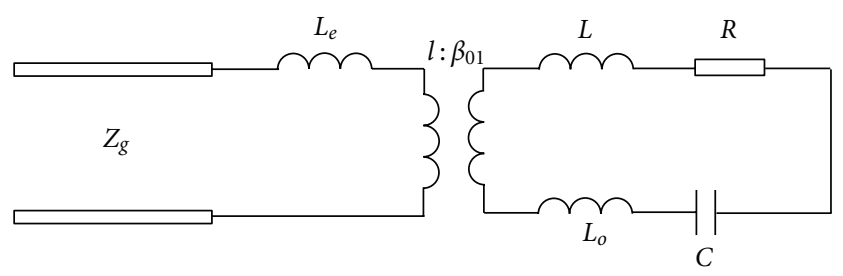

Figure 2: Equivalent circuit of open cavity fed by a rectangular waveguide.

From the circuit reported in Figure 2, it is straightforward to deduce the waveguide input impedance $Z_{i}$ and the reflection coefficient $\Gamma$, respectively, given as

$$
\begin{gathered}
Z_{i}=j \omega L_{e}+\frac{Z_{R}}{\beta_{01}^{2}}, \\
\Gamma=\frac{Z_{i}-Z_{10}}{Z_{i}+Z_{10}},
\end{gathered}
$$

where

$$
\begin{gathered}
Z_{R}=j \omega L_{T}+R^{\prime}+\frac{1}{j \omega C}, \\
L_{T}=L_{o}+L^{\prime} .
\end{gathered}
$$

In order to characterize the reflecting behavior of the single reflectarray element, a periodic array of identical elements is inserted at the center of the open cavity, as illustrated in Figure 3, where $R_{o}$ gives the radius of the spherical mirror and $w$ is the radius of the Gaussian beam. The open cavity configuration of Figure 1 results to be modified in Figure 3 by the insertion of a plane mirror at distance $l$, over which the microstrip array grid is fixed, thus half reducing the original open resonator system dimensions. As a consequence of this, the equivalent circuit of Figure 2 results to be modified as in Figure 4, with the addition of the impedance $Z_{a}$ taking into account the presence of the reflecting surface. It must be observed that, due to the presence of the metallic plane at the center of the cavity, only odd modes are admissible.

The insertion of the grounded reflecting surface can be modelled by the equivalent circuit of Figure 5 , where parameters $L_{1}, C_{1}$ depends on both the variable length $L$ of the 


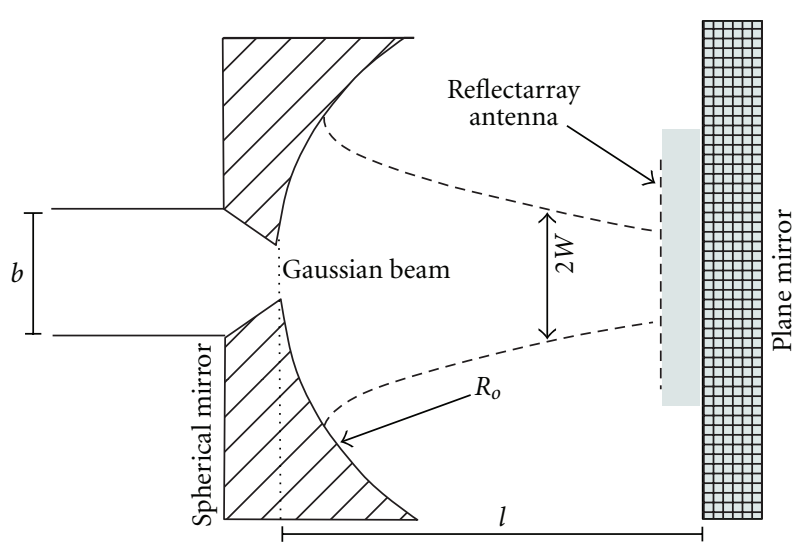

Figure 3: Open resonator system for reflectarray elements characterization.

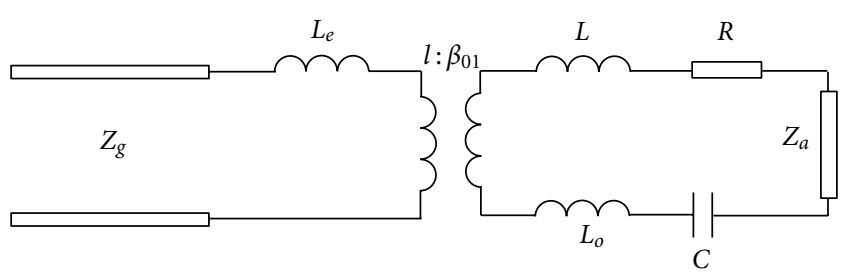

FIgURE 4: Equivalent circuit of open resonator system in Figure 3.

reflectarray patches and the grid spacing $D$ between adjacent elements.

Due to the small thickness $h$, usually less than a quarter wavelength, the impedance $Z_{\text {ind }}$ (Figure 5) can be simplified as follows:

$$
Z_{\text {ind }}=j Z_{d}\left(k_{d} h-\phi_{G}\right),
$$

where $Z_{d}=Z_{o} / \sqrt{\epsilon_{r}}, k_{d}=k_{o} \sqrt{\epsilon_{r}}, Z_{o}$ and $k_{o}$ being, respectively, the free-space impedance and propagation constant, while the phase shift $\phi_{G}$ takes into account the Gaussian nature of the beam $[27,28]$, and is given as

$$
\phi_{G}=\arctan \frac{h}{z_{R}} .
$$

The term $z_{R}=\sqrt{R l-l^{2}}$ into (4) represents the Rayleigh distance.

To simplify the treatment, without loss of generality, the assumption of lossless dielectric with a relative permittivity $\epsilon_{r}$ is made for the reflectarray surface. However, few additional derivations can be performed to treat the real loss case.

It is straightforward to recognize into expression (3) an inductive effect, which can be modelled in terms of an equivalent inductance $L_{u}=Z_{d}\left(k_{d} h-\phi_{G}\right)$, as reported in Figure 6.

When inserting the equivalent circuit of Figure 6 into that of Figure 4, the resonator will show two resonant frequencies $f_{1,2}$, relevant to the circuit of Figure 7, which are given as

$$
f_{1,2}=\frac{1}{2 \pi} \sqrt{\frac{c \pm \sqrt{c^{2}-4 d}}{2 d}}
$$

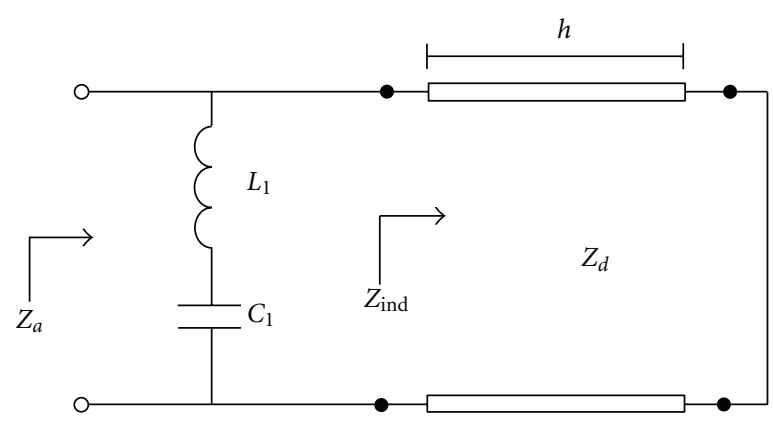

Figure 5: Equivalent circuit of grounded reflecting surface.

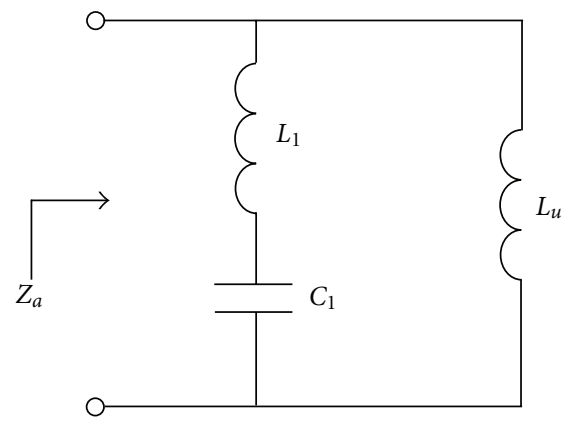

FIgURE 6: Simplification of equivalent circuit in Figure 5.

where

$$
\begin{gathered}
c=L_{o} C\left(L_{1}+L_{u}\right) C_{1}+C L_{1} L_{u} C_{1}, \\
d=L_{o} C+\left(L_{1}+L_{u}\right) C_{1}+L_{u} C .
\end{gathered}
$$

From expressions (6), the unknown circuit parameters $L_{1}, C_{1}$ can be easily derived as

$$
\begin{gathered}
L_{1}=\frac{C_{1} A_{1}+B_{1}}{C_{1} E_{1}}, \\
C_{1}=\frac{B_{2} E_{1}-B_{1} E_{2}}{A_{1} E_{2}-A_{2} E_{1}},
\end{gathered}
$$

where

$$
\begin{gathered}
A_{n}=\left(2 \pi f_{n}\right)^{2} L_{u}-\left(2 \pi f_{n}\right)^{4} L_{u} L_{o} C, \\
B_{n}=\left(2 \pi f_{n}\right)^{2}\left(L_{u}+L_{o}\right) C-1, \\
E_{n}=\left(2 \pi f_{n}\right)^{4}\left(L_{u}+L_{o}\right) C-\left(2 \pi f_{n}\right)^{2}
\end{gathered}
$$

for $n=1,2$.

The resonant frequencies $f_{1,2}$ are directly obtained from the return loss measurement at the feeding waveguide input of the open resonator. This is loaded with a reflecting surface made of identical square patches having size $L$ and spaced of a distance $D$. In order to best simulate the infinite array situation, a sufficient large array grid, typically greater than $7 \times 7$, must be considered.

As highlighted above, parameters $L_{1}, C_{1}$ are depending on the reflectarray geometry, so measurements need to be repeated for different patch dimensions in order to retrieve the phase response of the reflectarray element versus the tuning geometrical parameter. 


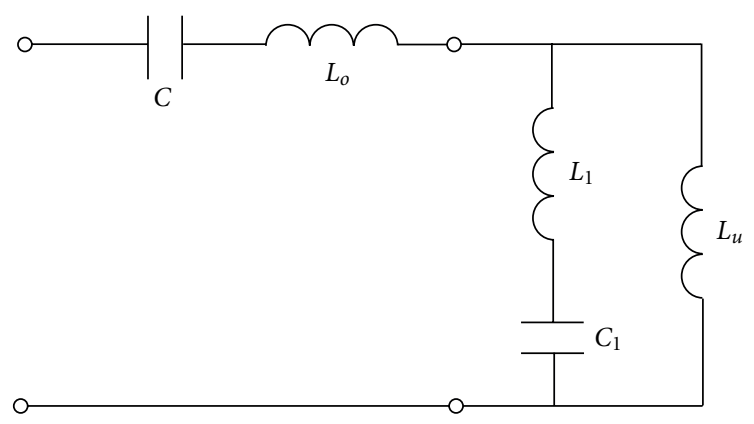

FIgURE 7: Resonant circuit for the computation of frequencies $f_{1,2}$.
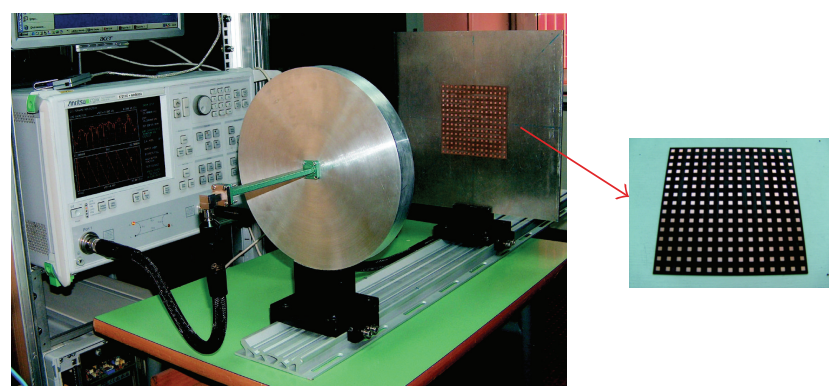

(a)

(b)

FIGURE 8: Photograph of (a) open resonator system and (b) realized reflecting surface.

\section{Experimental Validations}

In order to experimentally validate the method outlined in the previous section, a K-band open resonator is designed with a mirror radius $R_{o}=529 \mathrm{~mm}$ and a distance $l=$ $421 \mathrm{~mm}$ between the spherical and the flat mirrors. The resonator is tuned to work at a design frequency $f_{o}=24 \mathrm{GHz}$ (the center operating frequency of the reflectarray to be tested), which corresponds to the excitation of $\mathrm{TEM}_{0,0,131}$ mode inside the cavity.

The optimization of the waveguide-to-cavity transition is performed by following the approach outlined in [26], thus reducing the standard $W R G 2$ waveguide height $b=4.33 \mathrm{~mm}$ to a value of $0.7 \mathrm{~mm}$.

Reflectarray grids of $16 \times 16$ square patches with a spacing $D=0.65 \lambda_{o}$, for three different patch lengths $L(3.2 \mathrm{~mm}$, $3.5 \mathrm{~mm}$, and $3.7 \mathrm{~mm}$ ), are considered as test surfaces. A substrate dielectric with a nominal value $\epsilon_{r}=2.33$ and thickness $h=0.762 \mathrm{~mm}$ is considered. In Figure 8(a), a photograph showing the open resonator system loaded with the testing array is reported, while in Figure 8(b) one of the realized reflecting surface is illustrated.

The test setup is mounted into the Microwave Laboratory at University of Calabria, equipped with complete facilities for both nearfield and far-field measurements [29, 30].

The magnitude of the measured reflection coefficient at the waveguide input for the three different dimensions is illustrated in Figures 9-11. For all cases, several resonances couples are visible, corresponding to the various modes excited into the cavity. However, the only couple to be
TABLE 2: Resonant frequencies $f_{1,2}$ and relative values of parameters $L_{1}, C_{1}$.

\begin{tabular}{lcccc}
\hline Patch length $L(\mathrm{~mm})$ & $f_{1}(\mathrm{GHz})$ & $f_{2}(\mathrm{GHz})$ & $L_{1}(\mathrm{nH})$ & $C_{1}(\mathrm{fF})$ \\
\hline 3.2 & 23.95 & 25.88 & 4.06 & 7.55 \\
3.5 & 23.78 & 24.22 & 4.24 & 8.44 \\
3.7 & 22.95 & 24.02 & 4.54 & 8.70 \\
\hline
\end{tabular}

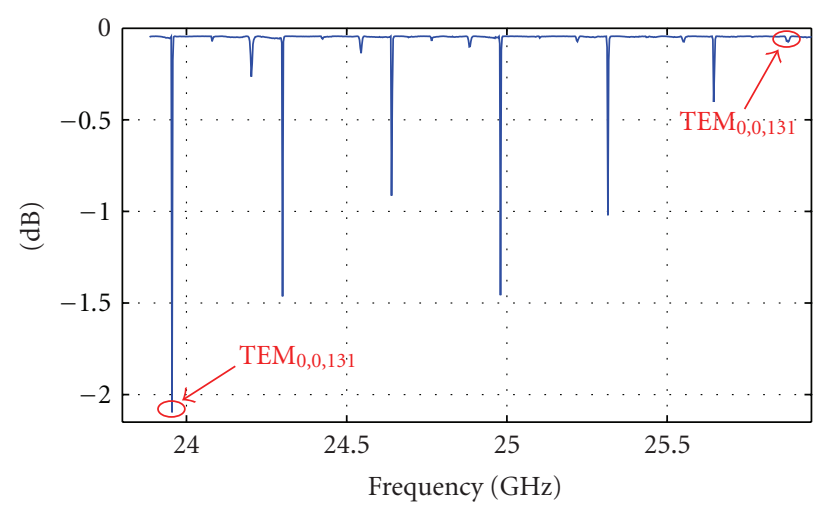

FIGURE 9: Measured return loss for the case $L=3.2 \mathrm{~mm}$.

considered is that modelled by the equivalent circuit of Figure 4 , corresponding to the $\mathrm{TEM}_{0,0,131}$ mode, which is associated to an empty cavity resonance $f_{o}=24 \mathrm{GHz}$. In the presence of each reflecting surface, two resonant frequencies $f_{1,2}$ are produced, as highlighted in the previous section. They can be easily identified in the return loss measurements (Figures 9-11) as follows.

(i) For a patch side dimension $L$ less than that providing the resonance condition, frequency $f_{1}$ is chosen as the nearest one (at the left side) to the resonance frequency $f_{o}$ of the empty cavity, while the frequency $f_{2}$ corresponds to the resonance of the reflectarray grid, easily computed on the basis of the patch dimension $L$. This is the case corresponding to Figure 9.

(ii) For a patch side dimension $L$ which is equal to that providing the resonance condition, frequencies $f_{1,2}$ are chosen as those which are equally far from the resonance frequency $f_{o}$ of the empty cavity. This is the case corresponding to Figure 10.

(iii) For a patch side dimension $L$ greater than that providing the resonance condition, frequency $f_{1}$ is chosen as the nearest one (at the right side) to the resonance frequency $f_{o}$ of the empty cavity, while the frequency $f_{2}$ corresponds to the resonant frequency of the reflectarray grid, again computed on the basis of the patch dimension $L$. This is the case corresponding to Figure 11.

The relevant resonances $f_{1,2}$ are highlighted in Figures 911 and summarized in Table 2.

The measured resonance frequencies $f_{1,2}$ are used to retrieve the values of parameters $L_{1}, C_{1}$ (Table 2), which are applied into the equivalent circuit of Figure 5 to compute 


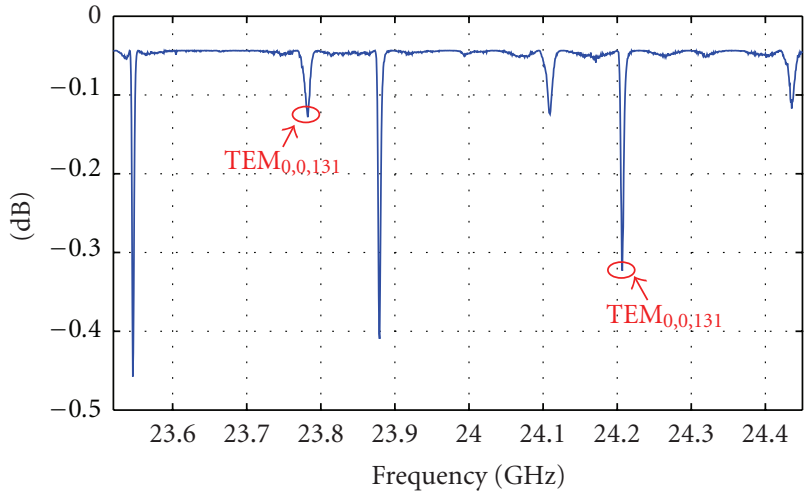

Figure 10: Measured return loss for the case $L=3.5 \mathrm{~mm}$.

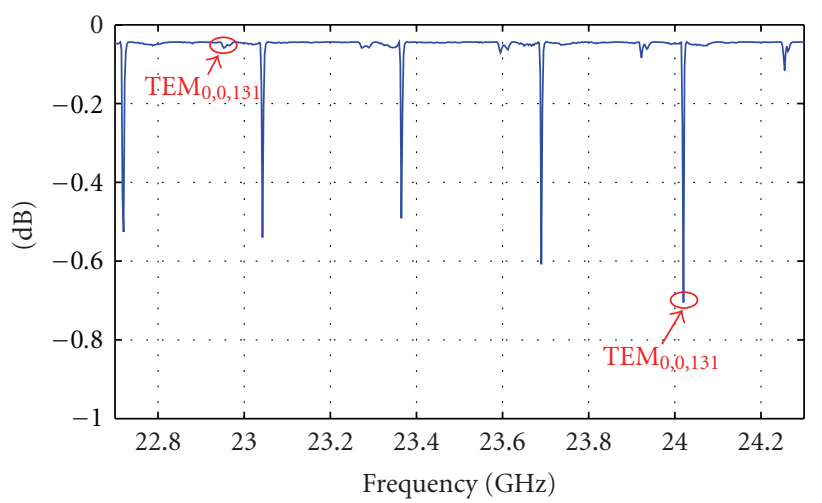

Figure 11: Measured return loss for the case $L=3.7 \mathrm{~mm}$.

the impedance $Z_{a}$, subsequently adopted in the circuit of Figure 4 to obtain the phase of the reflection coefficient relative to the reflectarray grid. Results are reported in Figures $12-14$ as a function of frequency for the three different dimensions of the patch side $L$. When performing the synthesis of microstrip reflectarrays [15], the reflection phase behavior of the single radiating element is of primary importance, as providing the proper dimensions of the tuning parameter (e.g., the patch side $L$ ) which are able to guarantee the phase distribution on the array grid assuring the prescribed-field pattern. To validate the results provided by the proposed approach, the reflection phase computed by Ansoft Designer software (infinite array approach) is also reported in Figures 12, 13, and 14, and a successful agreement can be observed.

Finally, the information retrieved from the three different measurements are combined to obtain the reflectarray phase design curve versus the patch length $L$. Again, the effectiveness of the approach is successfully demonstrated in Figure 15 by comparison with the results coming from Ansoft Designer simulations.

\section{Conclusions}

The use of an open cavity has been proposed in this work to characterize variable patches microstrip reflectarrays. An equivalent circuit including both the open resonator and the

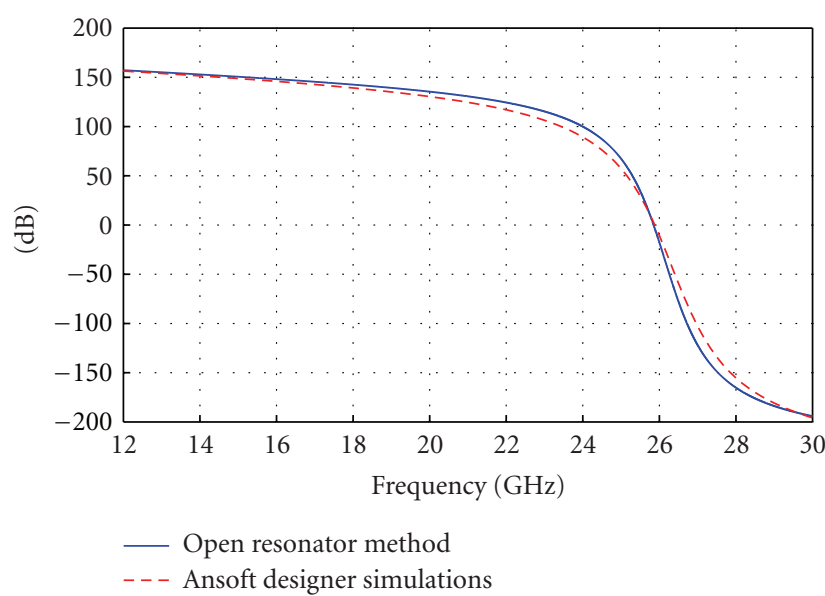

FIGURE 12: Reflection phase versus frequency for the case $L=$ $3.2 \mathrm{~mm}$.

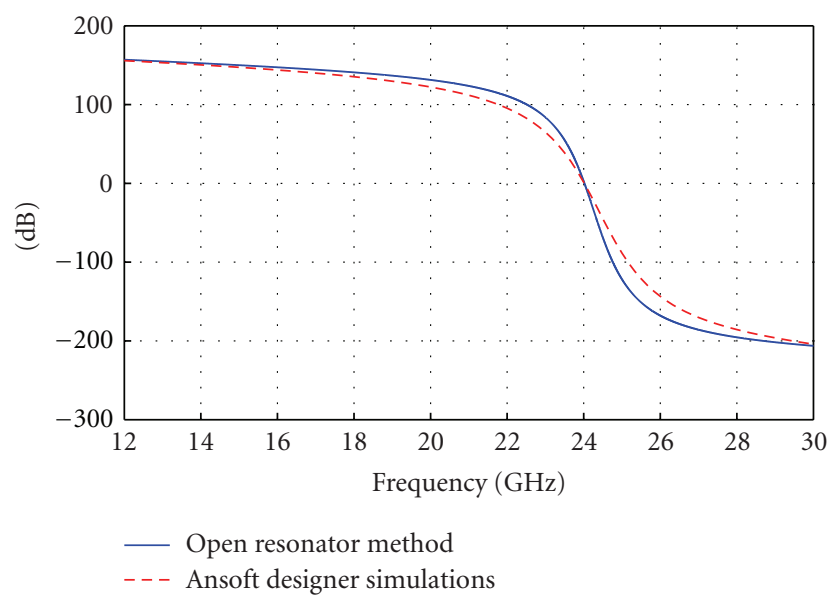

FIGURE 13: Reflection phase versus frequency for the case $L=$ $3.5 \mathrm{~mm}$.

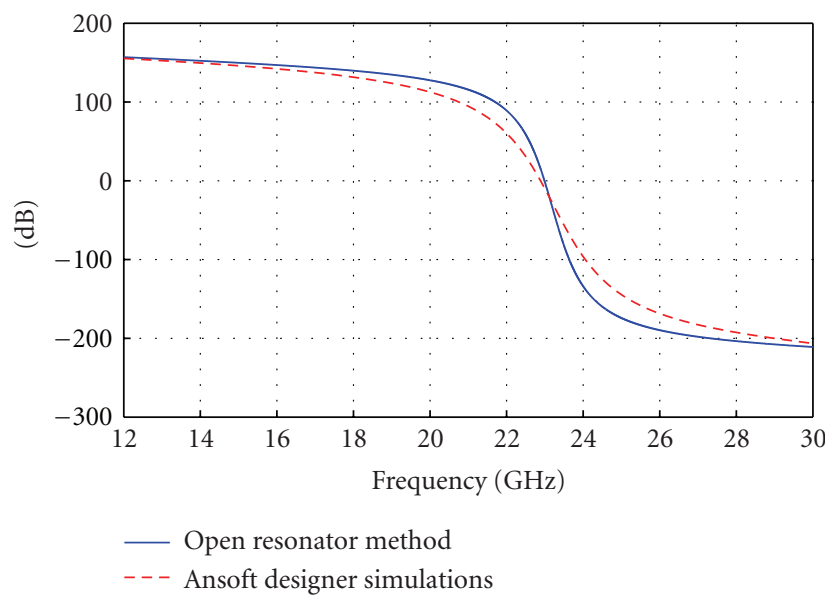

FIGURE 14: Reflection phase versus frequency for the case $L=$ $3.7 \mathrm{~mm}$. 


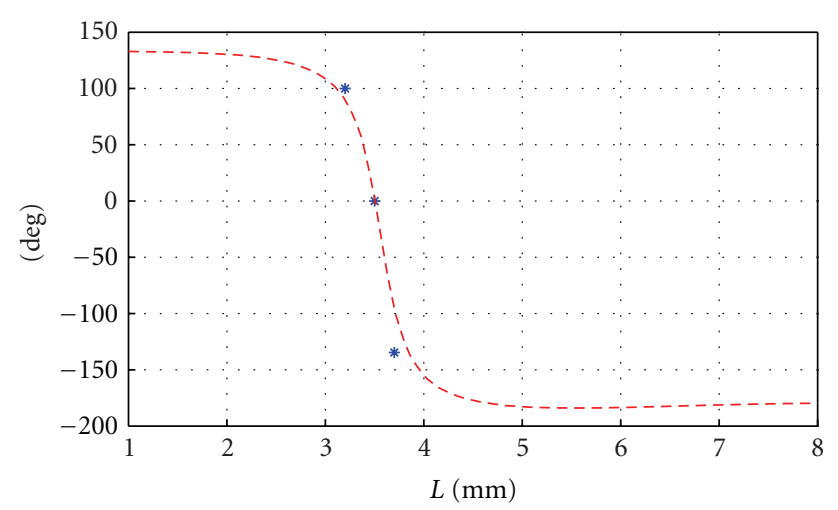

* Open resonator method

- - - Ansoft designer simulations

FIGURE 15: Reflection phase versus patch length $L$.

reflecting surface has been adopted to recover the reflection phase versus the patch length from return loss measurements at the feeding waveguide input. Experimental validations have been successfully discussed on K-band reflectarrays prototypes.

The proposed characterization method is particularly suitable for millimeter-wave applications [31,32], where the reduced dimensions [33] make impractical the adoption of standard techniques.

Concerning further developments, an extended formulation of the method including also the dielectric losses will be considered in future studies to fully characterize the reflectarray element response in both amplitude and phase.

\section{References}

[1] J. Huang and Analysis of a microstrip reflectarray antenna for microspacecraft applications, TDA Progress Report 153173, 1995.

[2] J. Huang, "Capabilities of printed reflectarray antennas," in Proceedings of the 1996 IEEE International Symposium on Phased Array Systems and Technology, pp. 131-134, October 1996.

[3] D. Berry, R. Malech, and W. Kennedy, "The reflectarray antenna," IEEE Transactions on Antennas and Propagation, vol. 11, no. 6, pp. 645-651, 1963.

[4] H. Fang, M. Lou, J. Huang, L.M. Hsia, and G. Kerdanyan, "Design and development of an inflatable refectarray antenna," IPN Progress Report 42-149, 2002.

[5] R. D. Javor, X. D. Wu, and K. Chang, "Design and performance of a microstrip reflectarray antenna," IEEE Transactions on Antennas and Propagation, vol. 43, no. 9, pp. 932-939, 1995.

[6] J. Huang and A. Encinar, Reflectarray Antennas, IEEE Press, NY, USA.

[7] D. M. Pozar, S. D. Targonski, and H. D. Syrigos, "Design of millimeter wave microstrip reflectarrays," IEEE Transactions on Antennas and Propagation, vol. 45, no. 2, pp. 287-296, 1997.

[8] J. C. Ginn, B. A. Lail, and G. D. Boreman, "Phase characterization of reflectarray elements at infrared," IEEE Transactions on Antennas and Propagation, vol. 55, no. 11 I, pp. 2989-2993, 2007.
[9] D. M. Pozar, S. D. Targonski, and R. Pokuls, "A shaped-beam microstrip patch reflectarray," IEEE Transactions on Antennas and Propagation, vol. 47, no. 7, pp. 1167-1173, 1999.

[10] A. Kelkar, "FLAPS: conformal phased reflecting surfaces," in Proceedings of the 1991 IEEE National Radar Conference, pp. 58-62, March 1991.

[11] J. Huang and R. J. Pogorzelski, "A ka-band microstrip reflectarray with elements having variable rotation angles," IEEE Transactions on Antennas and Propagation, vol. 46, no. 5, pp. 650-656, 1998.

[12] L. Boccia, F. Venneri, and G. Di Massa, "A Varactor Loaded Reflectarray Antenna," in Proceedings of the International Conference on Electromagnetics in Advanced Applications (ICEAA'01), September 2001.

[13] F. Venneri, S. Costanzo, G. Di Massa et al., "Beam-scanning reflectarray based on a single varactortuned element," International Journal of Antennas and Propagation, vol. 2012, Article ID 290285, 5 pages, 2012.

[14] F. Venneri, S. Costanzo, and G. Di Massa, "Reconfigurable aperturecoupled reflectarray element tuned by single varactor diode," Electronics Letters, vol. 48, pp. 68-69, 2012.

[15] F. Venneri, S. Costanzo, G. Di Massa, and G. Angiulli, "An improved synthesis algorithm for reflectarrays design," IEEE Antennas and Wireless Propagation Letters, vol. 4, no. 1, pp. 258-261, 2005.

[16] F. Venneri, S. Costanzo, G. Di Massa, P. Corsonello, and M. Salzano, "Design of a reconfigurable reflectarray based on a varactor tuned element," in Proceedings of the European Conference on Antennas and Propagation (EuCAP'12), Prague, Czech Republic, March 2012.

[17] S. V. Hum, M. Okoniewski, and R. J. Davies, "Modeling and design of electronically tunable reflectarrays," IEEE Transactions on Antennas and Propagation, vol. 55, no. 8, pp. 22002210, 2007.

[18] M. R. Chaharmir, J. Shaker, M. Cuhaci, and A. R. Sebak, "Novel photonically-controlled reflectarray antenna," IEEE Transactions on Antennas and Propagation, vol. 54, no. 4, pp. 1134-1141, 2006.

[19] R. C. Hansen, Phase Array Antennas, Wiley \& Sons, New York, NY, USA, 2009.

[20] F. C. E. Tsai and M. E. Bialkowski, "An equivalent waveguide approach to designing of reflect arrays with the use of variablesize microstrip patches," Microwave and Optical Technology Letters, vol. 34, no. 3, pp. 172-175, 2002.

[21] F. C. E. Tsai and M. E. Bialkowski, "Designing a 161-element $\mathrm{Ku}$-band microstrip reflectarray of variable size patches using an equivalent unit cell waveguide approach," IEEE Transactions on Antennas and Propagation, vol. 51, no. 10, pp. 29532962, 2003.

[22] J. C. Ginn, B. A. Lail, and G. D. Boreman, "Phase characterization of reflectarray elements at infrared," IEEE Transactions on Antennas and Propagation, vol. 55, no. 11 I, pp. 2989-2993, 2007.

[23] M. Bozzi, S. Germani, and L. Perregrini, "A figure of merit for losses in printed reflectarray elements," IEEE Antennas and Wireless Propagation Letters, vol. 3, no. 1, pp. 257-260, 2004.

[24] F. A. Tahir, Electromagnetic modeling of microstrip reflectarrays using scale changing technique [Ph.D. thesis], Universit di Toulouse, 2011.

[25] C. Fabry and A. Perot, "Theorie et applications d'une nouvlle method de spectroscopie interfrentielle," Annales de Chimie et de Physique, vol. 716, p. 115, 1899. 
[26] O. M. Bucci and G. Di Massa, "Open resonators powered by a rectangular waveguide," IEE Proceedings H: Microwaves, Antennas and Propagation, vol. 139, no. 4, pp. 323-329, 1992.

[27] Yu. P. K. and A. L. Cullen, "Measurement of permittivity by means of an open resonator - 1. theoretical," Proceedings of The Royal Society of London, Series A, vol. 380, no. 1778, pp. 49-71, 1982.

[28] A. C. Linch, "Measurement of permittivity by means of an open resonator. II Experimental," Proceedings of the Royal Society $A$, vol. 380, pp. 73-76, 1982.

[29] S. Costanzo and G. Di Massa, "An integrated probe for phaseless near-field measurements," Measurement, vol. 31, pp. 123129, 2002.

[30] S. Costanzo and G. Di Massa, "Direct far-field computation from bi-polar near-field samples," Journal of Electromagnetic Waves and Applications, vol. 20, pp. 1137-1148, 2006.

[31] S. Costanzo, I. Venneri, G. Di Massa, and G. Amendola, "Hybrid array antenna for broadband millimeter-wave applications," Progress In Electromagnetics Research-PIER, vol. 83, pp. 173-183, 2008.

[32] S. Costanzo, I. Venneri, G. Di Massa, and A. Borgia, "Benzocyclobutene as substrate material for planar millimeter-wave structures: dielectic characterization and application," Journal of Infrared, Millimeter and Terahertz Waves, vol. 31, pp. 66-77, 2010.

[33] S. Costanzo, "Synthesis of multi-step coplanar waveguide-tomicrostrip transition," Progress in Electromagnetics ResearchPIER, vol. 113, pp. 111-126, 2011. 

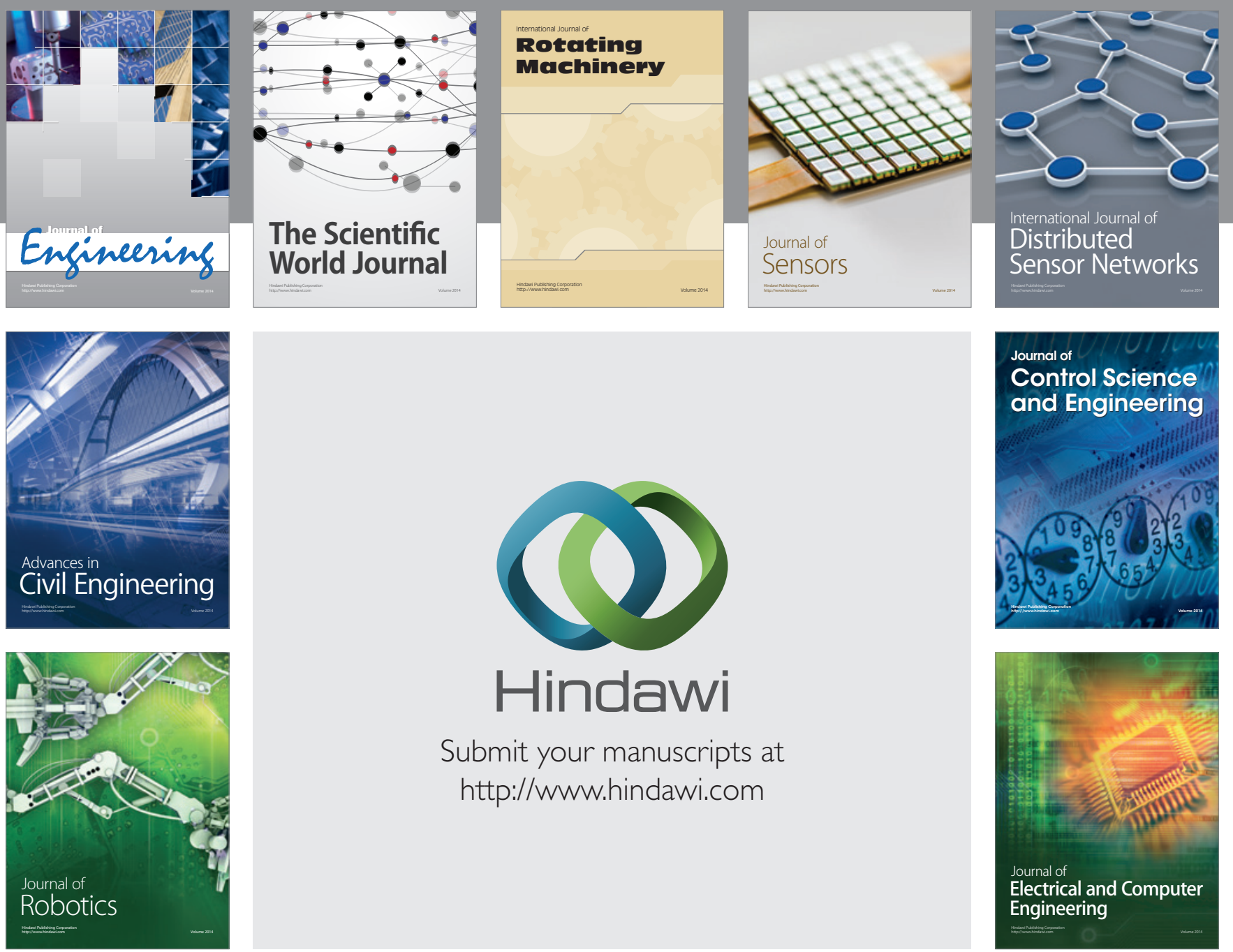

Submit your manuscripts at

http://www.hindawi.com
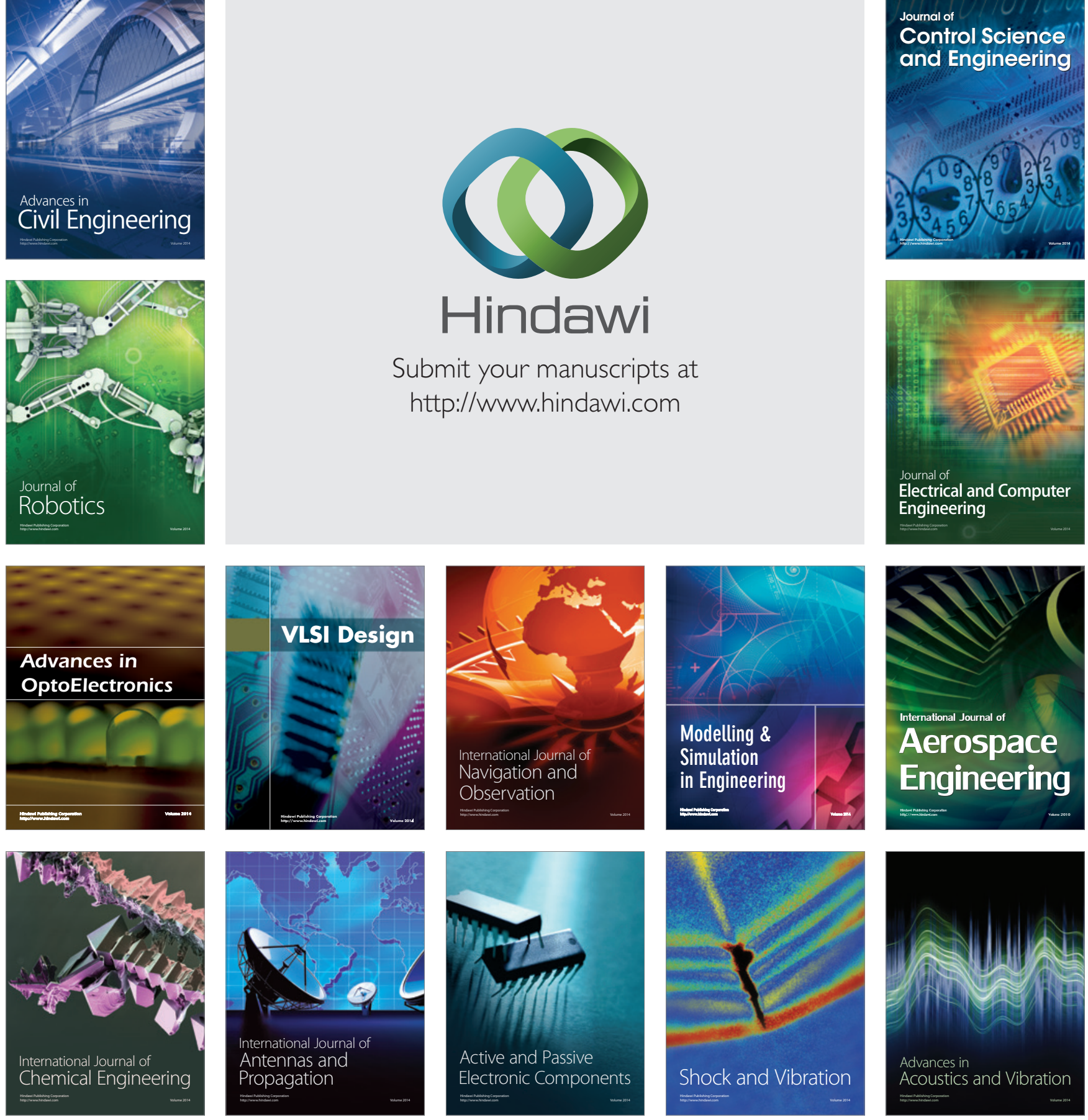